\title{
Heterojunctions Based on Amorphous Silicon: A Versatile Surface Engi- neering Strategy to Tune Peak Position of Redox Monolayers on Photo- electrodes
}

\author{
Vinicius R. Gonçales ${ }^{\text {abcs*, Jiaxin Lian }}{ }^{\text {abc§ }}$, Shreedhar Gautamª, Daniel Hagness ${ }^{a}$, Ying Yangabc, Richard \\ D. Tilley abd, Simone Ciampie, J. Justin Goodingabc* \\ a. School of Chemistry, The University of New South Wales, Sydney, NSW2052, Australia. \\ b. Australia Centre for NanoMedicine, The University of New South Wales, Sydney, NSW2052, Australia. \\ c. ARC Centre of Excellence in Convergent Bio-Nano Science and Technology, The University of New South Wales, \\ Sydney, NSW 2052, Australia. \\ d. Electron Microscopy Unit, Mark Wainwright Analytical Centre, The University of New South Wales, Sydney, \\ NSW2052, Australia. \\ e. School of Molecular and Life Sciences, Curtin Institute of Functional Molecules and Interfaces, Curtin University, \\ Bentley, WA6102, Australia. \\ *v.goncales@unsw.edu.au; justin.gooding@unsw.edu.au
}

\begin{abstract}
Heterojunctions are typically used to generate large photovoltages and to influence the direction of flow of charge carriers on photovoltaic and photocatalytic devices. Herein, we propose how heterojunctions can be used as a pathway for tuning the peak position of redox active monolayers. This was possible by exploring the principle of contact between materials in heterojunctions leading to a common equilibrium Fermi level for both sides of the heterojunction. The phenomenon was demonstrated with thin layers of intrinsic amorphous silicon deposited on platinum, indium tin oxide and either ntype or p-type crystalline silicon electrodes. At fixed light-intensity conditions, the potential required for electron transfer of a model redox probe was modulated according to the substrate on which the amorphous silicon was deposited. This allowed us to alter peak position of a redox process occurring on the electrolyte side of the junction despite it being isolated from the underlying conducting material. We show how such an effect can be explored in a potential range that encompasses any of the redox monolayers electroactive in aqueous electrolytes.
\end{abstract}

\section{INTRODUCTION}

Energetic aspects of charge-transfer across semiconductoraqueous electrolyte interfaces are of great importance to applications in energy conversion, ${ }^{1-2}$ chemical sensors, ${ }^{3-5}$ bioactive surfaces $^{6}$ and photocatalysis. ${ }^{7-11}$ Among semiconductors, industrially relevant silicon has proven a valuable material with the development of self-assembled monolayers based on $\alpha, \omega$-dialkynes, ${ }^{12}$ which are capable of avoiding anodic corrosion of silicon in aqueous electrolytes. ${ }^{13-14}$ Further derivatization of the alkyne-terminated monolayer via click-chemistry ${ }^{15}$ allows the design of applications according to the redox potential of the clicked electroactive molecules. ${ }^{3,16}$

In this context, for a given redox species attached to a photoactive silicon, modulation of peak positions was described possible as a function of light-intensity. This was attributed to alterations in the open circuit photovoltage caused by changes in the photogenerated current at the semiconductor|electrolyte junction. ${ }^{17-18}$ For instance, with increasing the light-intensity on a monolayer protected n-type silicon derivatized with ferrocene, both oxidation and reduction peaks gradually shift to more negative potentials in comparison to an electrode of metallic behavior. ${ }^{19}$ This is attributed to more electron-hole pairs being generated with the increase of light-intensity and as such the quasi-Fermi level of silicon moves to higher energy (more negative potentials) due to the progressive occupancy of the conduction band. In contrast, monolayer protected p-type silicon derivatized with anthraquinone had only the cathodic peak shifted with increasing illumination intensity. In this case, the quasiFermi level of silicon progressively moves to lower energy (more positive potentials). The explanation for the selectively tune of the cathodic peak relied on the fact that surface energetics associated to the electron-transfer from the conduction band had a more prominent influence than the holes transferred from the valence band. ${ }^{20}$

With the effectiveness of modulating the light-intensity to control the energetics of photoreactions on semiconductors, we propose a surface engineering strategy that allows one to modulate the peak position of redox monolayers without the need of changing illumination conditions. The concept is based on the use of heterojunctions, which are usually employed to generate large photovoltages and to influence the direction of flow of charge carriers on photovoltaic $^{21-25}$ and photocatalytic devices. ${ }^{26-27}$ The hypothesis tested here is based on the principle that contact of materi- 


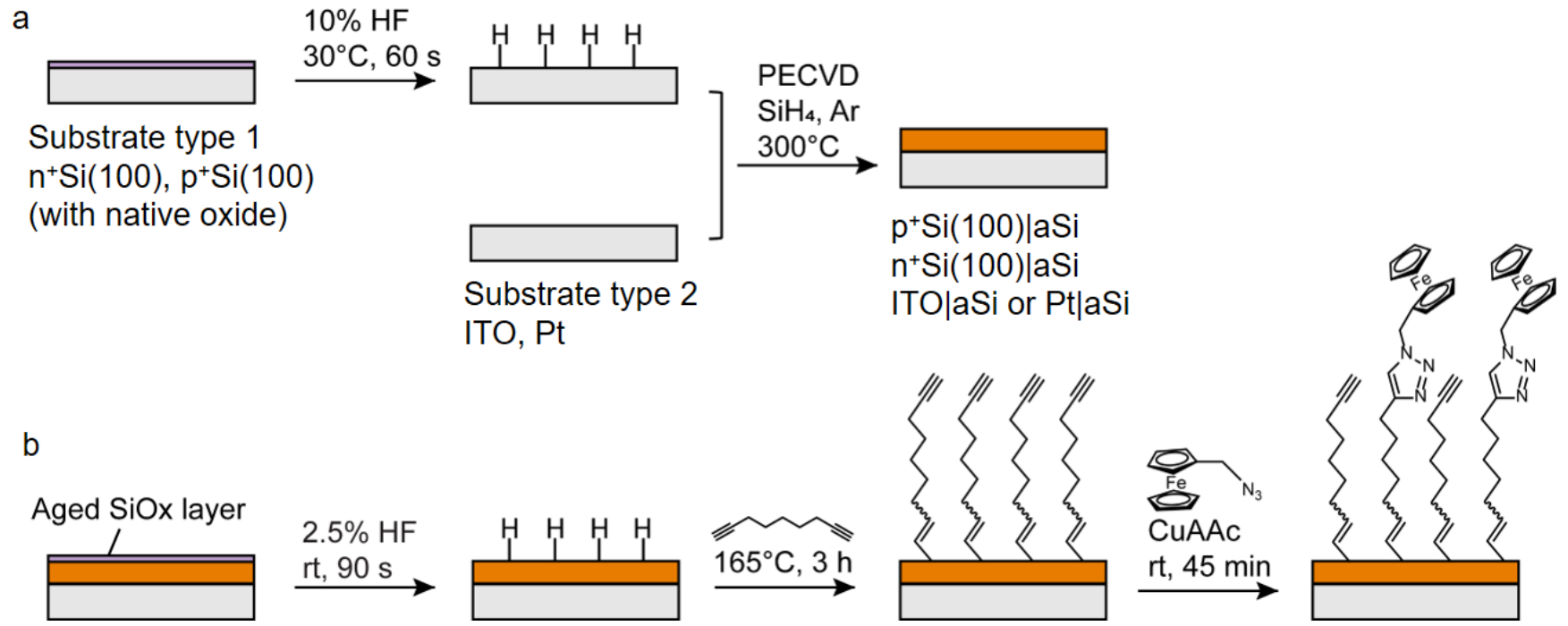

Scheme 1. (a) Modification of amorphous silicon-based heterojunctions with ferrocene redox probes. Intrinsic amorphous silicon was deposited by PECVD on four types of bulk electronic conductors: $\mathrm{n}$-type $\operatorname{Si}(100)(<0.007 \Omega \mathrm{cm}$, dopant concentration $\left.N_{D} \sim 8 \times 10^{18} \mathrm{~cm}^{-3}\right)$, p-type $\operatorname{Si}(100)\left(<0.003 \Omega \mathrm{cm}\right.$, dopant concentration $\left.\mathrm{N}_{\mathrm{A}} \sim 1 \times 10^{19} \mathrm{~cm}^{-3}\right)$, ITO and platinum. (b) For all electrodes, hydrogen-terminated amorphous silicon was initially reacted with 1,8-nonadiyne via thermal hydrosilylation.

als in heterojunctions tends to the creation of common equilibrium Fermi levels ( $\mathrm{E}_{\mathrm{F}}$ ) for both sides of the interface. ${ }^{28-29}$ Such heterojunctions enable the manipulation of surface Fermi energy without the introduction of dopants. ${ }^{30-31}$ From an electrochemical perspective, this principle indicates the possibility of tuning the potential required for electrontransfer of redox monolayers according to the materials used to form the heterojunction.

\section{METHODS}

\section{Chemicals and materials}

Purchased $\mathrm{H}_{2} \mathrm{O}_{2}$ (Sigma-Aldrich; 30 wt. \% solution), $\mathrm{H}_{2} \mathrm{SO}_{4}$ (J. T. Baker, 96 wt. \% solution) and HF (Riedel-de Haën, 48 wt. \% solution) were of semiconductor grade. All solvents used for cleaning and chemical modification procedures were distilled prior to use. 1,8-nonadiyne was redistilled from sodium borohydride (Sigma-Aldrich; $99 \%$ ) under reduced pressure $\left(65-70{ }^{\circ} \mathrm{C}, 25-30\right.$ Torr $)$ and stored under dry argon atmosphere prior to use. All aqueous electrolytes were of analytical grade. Milli-Q water $(>18 \mathrm{M} \Omega \mathrm{cm}$, Millipore, Australia) was used to prepare solutions.

Prime grade double-side polished n-type (phosphorous) silicon wafers, $(100) \pm 0.5^{\circ}, 175-225 \mu$ m thick, 8-12 $\Omega$ cm resistivity were purchased from Siltronix Silicon Technologies (SST, France). The dopant concentration was $\mathrm{N}_{\mathrm{D}} \sim$ $4.5 \times 10^{14} \mathrm{~cm}^{3}$ and the platform is referred here as lowlydoped n-type silicon ( $\left.\mathrm{n}^{-\mathrm{Si}}(100)\right)$. Prime grade single-side polished n-type (phosphorous) silicon wafers, (100) $\pm 0.5^{\circ}$, $500-50 \mu \mathrm{m}$ thick, $<0.007 \Omega \mathrm{cm}$ resistivity, $\mathrm{N}_{\mathrm{D}} \sim 8 \times 10^{18} \mathrm{~cm}^{3}$ is referred here as highly-doped n-type silicon ( $\left.n^{+} \operatorname{Si}(100)\right)$. Prime grade single-side polished p-type (boron) silicon wafers, $(100) \pm 0.5^{\circ}, 500-50 \mu \mathrm{m}$ thick, $<0.003 \Omega$ cm resistivity, $\mathrm{N}_{\mathrm{A}} \sim 1 \times 10^{19} \mathrm{~cm}^{3}$ is referred here as highly-doped p-type silicon $\left(\mathrm{p}^{+} \mathrm{Si}(100)\right)$. Prime grade single-side polished $\mathrm{p}$-type (boron) silicon wafers, $(100) \pm 0.5^{\circ}, 500-50 \mu \mathrm{m}$ thick, 1-10 $\Omega \mathrm{cm}$ resistivity, $\mathrm{N}_{\mathrm{A}} \sim 8 \times 10^{14} \mathrm{~cm}^{3}$ is referred here as lowlydoped p-type silicon ( $\mathrm{p}$-Si(100)). Platinum foil, $0.05 \mathrm{~mm}$ thick, $99.99 \%$ trace metals basis was purchased from Sigma-Aldrich. ITO coated coverslip (18 x $18 \mathrm{~mm}$, coverslip thickness \# 1.5, 8 - $12 \Omega$ resistivity) was purchased from SPI Supplies.

\section{Preparation of amorphous silicon-based heterojunctions}

The preparation of amorphous silicon-based heterojunctions is summarized in Scheme $1 \mathrm{a}$. Si(100) wafers were rinsed with dichloromethane, dried under a stream of argon, and immersed in Piranha solution (3:1 v/ $/ \mathrm{v} \mathrm{H}_{2} \mathrm{SO}_{4}: \mathrm{H}_{2} \mathrm{O}_{2}$ ) for $10 \mathrm{~min}$. Samples were then rinsed with Milli-Q water for 10 min before being transferred to $\mathrm{HF}$ aqueous solution $\left(1: 10 \mathrm{v} / \mathrm{v} \mathrm{HF}: \mathrm{H}_{2} \mathrm{O} ; \mathrm{T}=30^{\circ} \mathrm{C}\right)$ for $1 \mathrm{~min}$. Finally, the crystalline Si-H wafers were rinsed with Milli-Q water for $1 \mathrm{~min}$. Intrinsic amorphous silicon was then deposited using an Oxford Instruments Plasmalab 100 plasma-enhanced chemical vapor deposition system (PECVD). $\mathrm{SiH}_{4} 25 \mathrm{sccm}$ (Coregas, 99.999\%) was used as the precursor and Ar 475 sccm (Coregas, 99.99999\%) was employed as the carrier. Plasma was generated by using a $13.56 \mathrm{MHz}$ RF generator. Deposition of amorphous silicon was performed at $15 \mathrm{~W}$, 1000 mTorr, $300{ }^{\circ} \mathrm{C}$ until the aimed thickness was reached.

Platinum foils were rinsed with dichloromethane, dried under a stream of argon, and immersed in Piranha solution for $10 \mathrm{~min}$. Samples were then rinsed with Milli-Q water for 10 min before deposition of amorphous silicon at the same conditions specified for the silicon wafers.

ITO coated coverslips were sonicated in absolute ethanol for $10 \mathrm{~min}$ and in $0.5 \mathrm{M} \mathrm{K}_{2} \mathrm{CO}_{3}$ solution prepared with 3:1 $\mathrm{v} / \mathrm{v}$ methanol: $\mathrm{H}_{2} \mathrm{O}$ for $30 \mathrm{~min}$. ITO was rinsed with a large amount of Milli-Q water, then with acetone and isopropanol, before drying. They were then submitted to plasma cleaning 
for $10 \mathrm{~min}$. Prior to the deposition of amorphous silicon, a small piece of Si wafer (about $3 \mathrm{~mm} \times 3 \mathrm{~mm}$ ) was placed at one corner of the ITO coverslip to block the amorphous silicon deposition at that site. This is for preserving a connection point to the external circuit for the electrochemical measurements. Deposition of amorphous silicon was performed at $20 \mathrm{~W}, 1500 \mathrm{mTorr}, 300^{\circ} \mathrm{C}$ until the aimed thickness was reached. For ITO, He $475 \mathrm{sccm}$ was used as the carrier gas. $^{32}$

\section{Click-attachment of redox probes onto alkyne-terminated amorphous silicon}

Azidomethylferrocene was synthesized from commercially available ferrocenemethanol according to a previously reported procedure. ${ }^{33}$ The redox species 2-(azidomethyl)anthracene-9,10-dione was synthesized in one step from 2(bromomethyl)anthracene-9,10-dione. ${ }^{20}$

The $\mathrm{pSi}(100)|\mathrm{aSi}, \mathrm{nSi}(100)| \mathrm{aSi}$ and $\mathrm{Pt} \mid \mathrm{aSi}$ electrodes were washed with dichloromethane, dried under a stream of argon, and immersed in Piranha solution (3:1 v/ $\mathrm{v} \mathrm{H}_{2} \mathrm{SO}_{4}: \mathrm{H}_{2} \mathrm{O}_{2}$ ) for $30 \mathrm{~min}$. Samples were then rinsed with Milli-Q water before being transferred to $\mathrm{HF}$ (2.5 wt. \% aqueous solution) for $90 \mathrm{~s}$ to remove the aged silicon oxide layer and generate a hydrogen-terminated surface on amorphous silicon. ITO|aSi platforms were washed with redistilled ethanol and subjected to plasma cleaning for $1 \mathrm{~h}$ to remove any organic contaminants on the surface before HF treatment. This short etching process is negligibly deleterious to the glass substrate, but care was taken for not exposing the uncoated ITO corner to HF. After that, the amorphous silicon-based electrodes were immediately immersed into deoxygenated 1,8-nonadiyne in order to form the alkyne-terminated selfassembled monolayer, as depicted in Scheme 1b. Deoxygenation occurred in a Schlenk flask for a minimum of five freeze-pump-thaw cycles performed under 60 mTorr. The thermal hydrosilylation was performed under UHP argon atmosphere for $3 \mathrm{~h}$ into an oil bath set to $165^{\circ} \mathrm{C}$. After cooling to room temperature, the alkyne-terminated amorphous silicon surface was rinsed several times with dichloromethane and kept still in this solvent at $+4{ }^{\circ} \mathrm{C}$ under argon for 12 h. ${ }^{15}$

The alkyne-terminated surfaces were derivatized with azidomethylferrocene by placing samples in reaction vials. To the vials, it was added (i) $10 \mathrm{mM}$ azidomethylferrocene in isopropanol, (ii) $100 \mathrm{mM}$ sodium ascorbate in water and (iii) $0.4 \mathrm{mM}$ copper sulphate in water with the volume ratio of $4: 1: 1$, respectively. The reactions were carried out for 45 min at room temperature under argon atmosphere in the dark. Surfaces were then rinsed consecutively with copious amount of isopropanol, ethanol, $0.5 \mathrm{M} \mathrm{HCl}$ and then Milli-Q water prior to analyses. ${ }^{34}$

For the mixed redox self-assembled monolayer shown in Figure 6, the alkyne-terminated surfaces were half-coated with a photomask based on a SU-8 2007 photoresist (Microchem). For that, the photoresist was spin-coated at $65^{\circ} \mathrm{C}$ for 2 min followed by $3 \mathrm{~min}$ at $95^{\circ} \mathrm{C}$. For the patterning, the SU82007 photoresist was exposed to UV light for $22 \mathrm{~s}$ under a photomask that covered half of the surface (Quintel Q6000 Mask Aligner). The process was followed by repeating the baking step. The sample was then developed by immersing the amorphous silicon-based surfaces in SU-8 2007 developer (Microchem) for $2 \mathrm{~min}$, rinsed in isopropyl alcohol and dried under nitrogen flow. The SU-8 2007 photoresist located in regions not exposed to UV light was then washed away from the surface, resulting in the formation of the photoresist pattern that coated $50 \%$ of the area. Azidomethylferrocene was clicked on the exposed alkyne-terminated surfaces as previously described. Then, the SU-8 2007 photoresist was removed with DCM and the revealed alkyne-terminated surface was used to click 2-(azidomethyl)anthracene-9,10-dione. This was also done via click $\mathrm{Cu}(\mathrm{I})$-catalyzed azide-alkyne cycloaddition. For that, the amorphous silicon based surfaces were placed in a dimethyl sulfoxide solution containing $2 \mathrm{mM} 2$-(azidomethyl)anthracene-9,10-dione, 9.76 mM copper-(I) bromide, and $20.6 \mathrm{mM}$ tetramethylethylenediamine. ${ }^{20}$ Reactions were carried out at room temperature for 20 min under argon environment. The prepared silicon samples were rinsed consecutively with copious amounts of water, ethanol, and dichloromethane.

\section{X-ray photoelectron spectroscopy}

X-ray photoelectron spectroscopy (XPS) characterization was performed with an ESCALab $250 \mathrm{Xi}$ (Thermo Scientific) spectrometer with a monochromated $\mathrm{Al} \mathrm{K}_{\alpha}$ source. The pressure in the analysis chamber during measurements was $<10^{-8} \mathrm{mbar}$. The pass energy and step size for narrow scans were $20 \mathrm{eV}$ and $0.1 \mathrm{eV}$ respectively, with a take-off angle normal to the sample surface. Spectral analysis was performed by using the Avantage 4.73 software and curve fittings were carried out with a mixture of Gaussian-Lorentzian functions. Peaks were calibrated to C-C at $284.8 \mathrm{eV}$.

\section{Electrochemical measurements}

Cyclic voltammetry measurements were performed using a CHI660D potentiostat. A conventional three-electrode cell was used with $\mathrm{Ag}|\mathrm{AgCl}| 1 \mathrm{M} \mathrm{KCl}$ as reference electrode, a platinum mesh as counter electrode and the modified amorphous silicon-based heterojunctions as working electrodes. Ohmic contact to the pSi(100)|aSi, nSi(100)|aSi and Pt|aSi electrodes was achieved by rubbing a Ga-In eutectic mixture onto the scratched backside of the Si(100) electrodes and pressing it against a copper plate. On the ITO|aSi, the electronic contact was done on the exposed ITO corner. A white lamp ( $94 \mathrm{~mW} \mathrm{~cm}^{-2}$ ) was used as the light source to illuminate the top side of the amorphous silicon-based substrates.

\section{RESULTS AND DISCUSSION}

Three types of heterojunctions were prepared to evaluate the influence of the underlying semiconductor in a semiconductor-amorphous silicon heterojunction on the peak position of a ferrocene redox probe attached to the junction as in Scheme 1. This was achieved by depositing a $200 \mathrm{~nm}$ layer of intrinsic amorphous silicon onto: highly doped ntype Si(100), highly-doped p-type Si(100) and indium tin oxide. These electrodes are referred hereafter as $\mathrm{n}^{+} \mathrm{Si}(100)\left|\mathrm{aSi}, \mathrm{p}^{+} \mathrm{Si}(100)\right| \mathrm{aSi}$ and ITO|aSi, respectively. For all cases, the underneath substrate acts as the electronic 
bulk conductor for the photoactive amorphous layer. The rationality of combining these materials is justified in Scheme 2, which shows the energy levels associated with each semiconductor prior to the formation of the heterojunctions. When forming a heterojunction of two semiconductors, when the isolated semiconductors are brought in contact, electrons can move from the semiconductor with the higher Fermi level to the other, and an electric field is produced to balance this transfer. ${ }^{29}$ Under thermal equilibrium conditions, the effect tends to create a common equilibrium Fermi level for both semiconductors and generates an in-built junction potential that reflects the difference in work functions of each semiconductor. ${ }^{35}$ As shown in Scheme 2, the $\mathrm{E}_{\mathrm{F}}$ value associated with $\mathrm{n}^{+} \mathrm{Si}(100)$ is located at more negative potentials than the $\mathrm{E}_{\mathrm{F}}$ value associated with intrinsic aSi, whilst $\mathrm{p}^{+} \mathrm{Si}(100)$ displays an $\mathrm{EF}_{\mathrm{F}}$ value at more positive potentials than aSi. ITO is an intermediate case, as its Fermi level is located at similar energies than intrinsic aSi.

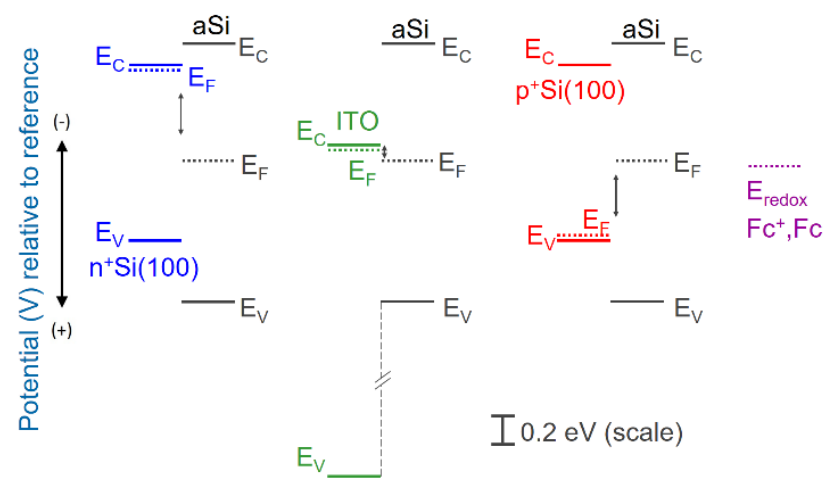

Scheme 2 - Band diagrams describing the interfaces prior to the formation of the amorphous silicon-based heterojunctions. Energy levels referenced to vacuum level according to Table S1.

From an electrochemical perspective, the principle suggests that when the amorphous silicon and the ferrocene are brought in contact in the absence of light, the energy level in which the electrostatic equilibrium ${ }^{36-37}$ is achieved between both phases will also depend on the heterojunction. This electrostatic equilibrium relies on charge transfer between unlevelled $\mathrm{E}_{\text {redox }}$ (from ferrocene/ferricenium) and $\mathrm{E}_{\mathrm{F}}$ (from amorphous silicon) energy values. Because $E_{F}$ value of the thin amorphous silicon layer is affected by charge carriers exchanged with the underneath bulk conductor, the open circuit photovoltage established under illumination will also become influenced by the substrate on which amorphous silicon is deposited. Therefore, under constant illumination, it is possible to alter the peak position of a given redox process occurring on the electrolyte side of the junction despite it being isolated from the underlying conducting material.

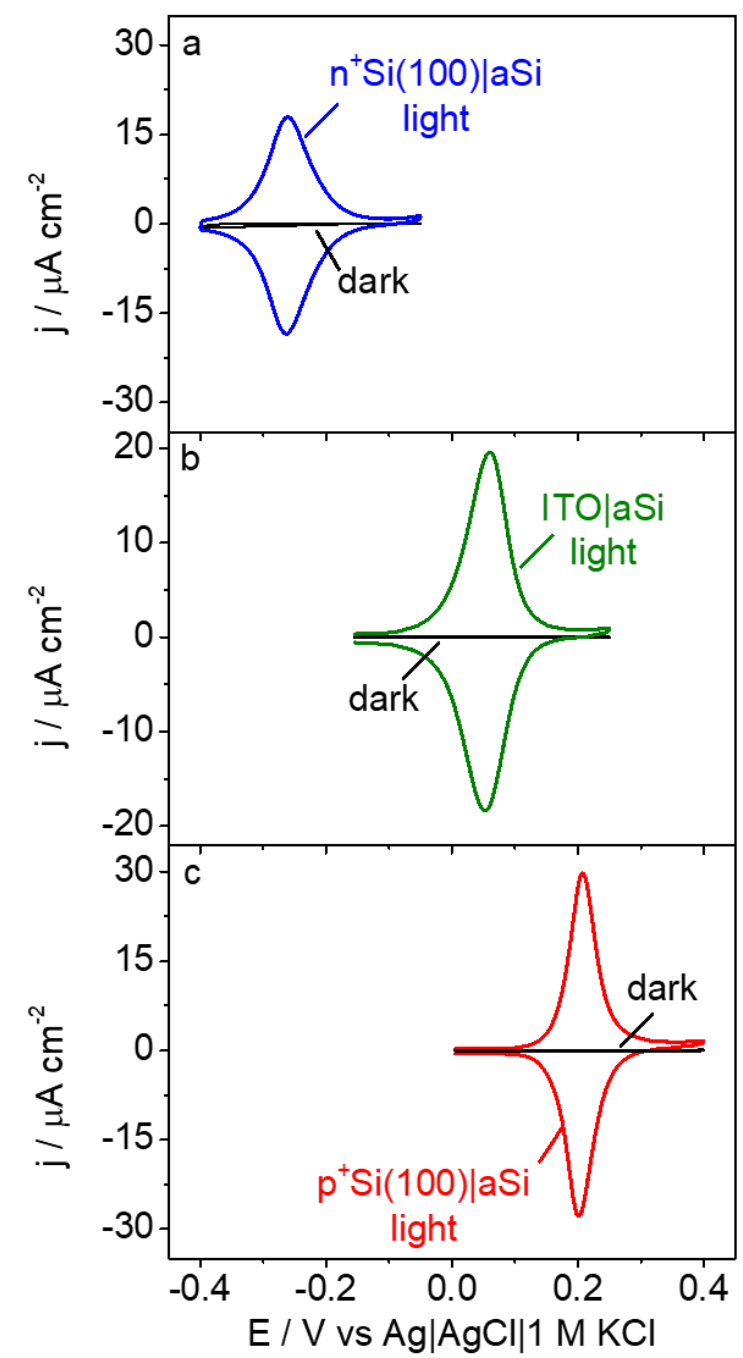

Figure 1 - Cyclic voltammograms of a ferrocene redox probe attached onto the monolayer-protected amorphous silicon. Photoactive amorphous silicon (thickness $=200 \mathrm{~nm}$ ) deposited onto (a) highly-doped n-type Si(100), (b) ITO and (c) highly-doped p-type $\mathrm{Si}(100)$. Electrolyte $=1 \mathrm{M} \mathrm{HClO}_{4} ; v=$ $100 \mathrm{mV} \mathrm{s}^{-1}$; surface topside illuminated with supra bandgap white light at $94 \mathrm{~mW} \mathrm{~cm}^{-2}$.

In order to demonstrate this possibility, thermal hydrosilylation with 1,8-nonadiyne was performed to provide hydrogen-terminated amorphous silicon with the stability needed to serve as a photoelectrode in aqueous media. ${ }^{32,38}$ A redox ferrocene probe was grafted to the distal end of the 1,8-nonadiyne via $\mathrm{Cu}(\mathrm{I})$-catalyzed azide-alkyne-cycloaddition reaction (Figure S1). ${ }^{33}$ As shown in the cyclic voltammograms in Figure 1, no notable faradaic current was detected in the dark for ferrocene-modified ${ }^{+} \mathrm{Si}(100) \mid \mathrm{aSi}$, $\mathrm{p}^{+} \mathrm{Si}(100) \mid \mathrm{aSi}$ and ITO|aSi. When a supra-bandgap light was employed to illuminate the surfaces (aSi bandgap $\sim 1.7$ $\mathrm{eV})^{39}$, both oxidation and reduction waves of ferrocene were observed. Even though the amorphous silicon|redox SAM interface was identical for all functionalized electrodes, the measured $\mathrm{E}_{1 / 2}$ values were affected by the bulk conductor on which amorphous silicon was deposited. The $\mathrm{E}_{1 / 2}$ values were $-0.261 \mathrm{~V}$ for $\mathrm{n}^{+} \mathrm{Si}(100) \mid \mathrm{aSi},+0.057 \mathrm{~V}$ for 
ITO $\mid \mathrm{aSi}$ and $+0.204 \mathrm{~V}$ for $\mathrm{p}^{+} \mathrm{Si}(100) \mid \mathrm{aSi}$ (vs $\mathrm{Ag}|\mathrm{AgCl}| 1 \mathrm{M}$ $\mathrm{KCl})$. A representation of the band diagrams associated with $\mathrm{n}^{+} \mathrm{Si}(100)\left|\mathrm{aSi}, \mathrm{p}^{+} \mathrm{Si}(100)\right| \mathrm{aSi}$ and ITO|aSi can be found in Scheme 3. The localized electronic states available into the amorphous layer bandgap originates from unpaired electrons in distorted $\mathrm{sp}^{3}$ hybrid orbitals. ${ }^{40}$

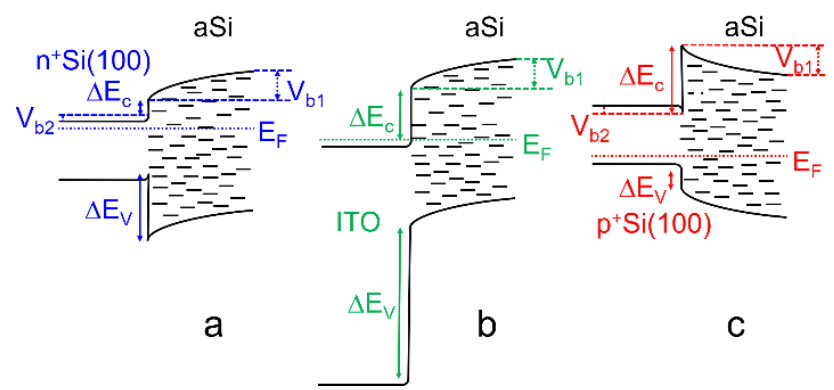

Scheme 3 - Representation of the band diagrams associated to (a) $n^{+} \operatorname{Si}(100) \mid a S i$, (b) ITO $\mid a S i$ and (c) $p^{+} \operatorname{Si}(100) \mid a S i$ heterojunctions. After formation of the heterojunction, the transition at the interface between the two semiconductors causes energy discontinuities on the conduction and valence bands, $\Delta \mathrm{E}_{\mathrm{c}}$ and $\Delta \mathrm{E}_{\mathrm{v}}$. The band discontinuities may be expressed as $\Delta \mathrm{E}_{\mathrm{C}}=\mathrm{EC}_{1}-\mathrm{EC}_{2}-\mathrm{eV}_{\mathrm{bi}}$ and $\Delta \mathrm{E}_{\mathrm{V}}=\mathrm{Eg}_{1}-\mathrm{Eg}_{2}-\Delta \mathrm{E}_{\mathrm{C}}$, where $\mathrm{EC}_{1}$ and $\mathrm{EC}_{2}$ are relative positions of the edges of the conduction bands of the two materials with respect to the Fermi level, $\mathrm{E}_{\mathrm{g} 1}$ and $\mathrm{E}_{\mathrm{g} 2}$ are the bandgaps and $\mathrm{V}_{\mathrm{bi}}$ is the builtin voltage at the heterojunction. The in-built voltage is given by $V_{b i}=V_{b 1}+V_{b 2}+\Delta \varphi_{i n}$, where $V_{b 1}$ and $V_{b 2}$ are the bandbending voltages on each side of the interface, and $\Delta \varphi_{\text {in }}$ is the potential step due to dipoles at the heterojunction

Further evidence for the influence of the bulk conductor on the thin amorphous silicon layer is shown in Figure S2. Figure $\mathrm{S} 2 \mathrm{a}$ shows how $\mathrm{E}_{1 / 2}$ shifts when highly-doped $\mathrm{Si}(100)$ is replaced by lowly-doped $\mathrm{Si}(100)$ as the bulk electronic conductor on which the intrinsic amorphous layer is deposited. At constant temperature, the position of the Fermi level of a n-type silicon is displaced to the anodic direction by diminishing the donor concentration. For a p-type silicon, the Fermi level position is displaced in the cathodic direction by diminishing the concentration of acceptors. These trends are followed by the ferrocene $\mathrm{E}_{1 / 2}$ positions. Additionally, Figure $\mathrm{S} 2 \mathrm{~b}$ shows cyclic voltammograms of ferrocene redox species attached to an insulating glass|intrinsic amorphous silicon junction. No electroactivity was observed either in the dark or under illumination with the glass- amorphous silicon junction. These results corroborate that charge exchange with the underlying substrate is essential to modulate electrochemistry on the amorphous silicon-based heterojunctions.

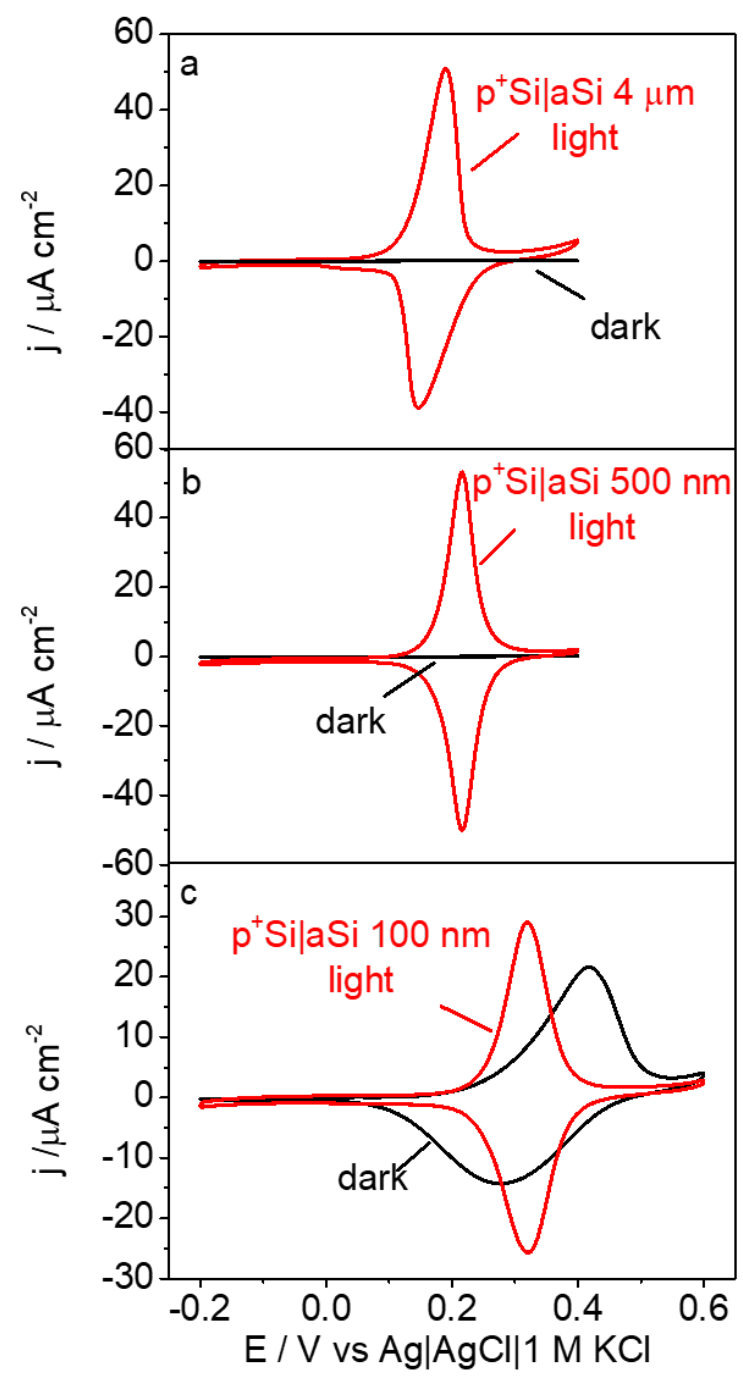

Figure 2 - Cyclic voltammograms of a ferrocene redox probe attached onto the monolayer-protected amorphous silicon. Photoactive amorphous silicon deposited onto highlydoped p-type Si(100) with thicknesses of (a) $4 \mu \mathrm{m}$, (b) 500 $\mathrm{nm}$ and (c) $100 \mathrm{~nm}$. Electrolyte $=1 \mathrm{M} \mathrm{HClO}_{4} ; \nu=100 \mathrm{mV} \mathrm{s}^{-1}$; surface topside illuminated with supra bandgap white light at $94 \mathrm{~mW} \mathrm{~cm}^{-2}$.

The effect of the thickness of the amorphous silicon layer on the electroactivity of ferrocene is a relevant parameter to be evaluated here. This is because the mechanism of chargecarriers transport on amorphous silicon is different from the crystalline silicon. On crystalline silicon, charge carriers can travel through extended electronic states. On amorphous silicon, due to the presence of localized electronic states into the bandgap (Scheme 3), hopping conduction through a multistep tunneling process also takes place. ${ }^{41}$ The effect is shown for a $\mathrm{p}^{+} \mathrm{Si}(100) \mid \mathrm{aSi}$ heterojunction in Figure 2. When a $4 \mu \mathrm{m}$ layer of amorphous silicon is deposited (Figure 2a), a pathway of high-resistance is created for charge-carriers to be transferred between ferrocene and the $\mathrm{p}^{+} \mathrm{Si}(100)$ bulk conductor. The consequence of a relatively thick amorphous silicon layer is seen on the unmatched peak currents and on the observed ohmic slope. By diminishing the thickness of the amorphous silicon layer to 
$500 \mathrm{~nm}$ (Figure 2b), the peak position of ferrocene/ferricenium $\left(\mathrm{E}_{1 / 2}=+0.216 \mathrm{~V}\right)$ is similar to the result reported in Figure 1 for a $200 \mathrm{~nm}$ layer $\left(\mathrm{E}_{1 / 2}=+0.204 \mathrm{~V}\right)$. The situation changes with a $100 \mathrm{~nm}$ amorphous silicon layer, where $E_{1 / 2}$ was found to be $+0.321 \mathrm{~V}$ and closer to the $+0.360 \mathrm{~V}$ value reported on $\mathrm{p}^{+} \mathrm{Si}(100)$ without an amorphous silicon layer. ${ }^{42}$ This behavior in Figure $2 \mathrm{c}$ is attributed to the ineffectiveness of the thin amorphous layer from blocking the dark communication between ferrocene tethers and $\mathrm{p}^{+} \operatorname{Si}(100)$. That is, in the case of very thin layers of amorphous silicon, the heterojunctions begin to behave electrochemically in a similar manner to the underlying bulk conductor. However, the phenomenon is dependent on the materials forming the heterojunction. As shown in Figure S3, for the ITO|aSi heterojunction, only $20 \mathrm{~nm}$ of amorphous silicon is enough to prevent dark communication with ferrocene. ${ }^{32}$

To demonstrate the scope of the methodology, heterojunctions were also formed by depositing intrinsic amorphous silicon on platinum (Figure 3 ). In this case, a much thicker amorphous silicon layer of $500 \mathrm{~nm}$ was required to block dark communication with ferrocene. This indicates the existence of a large dark leakage current across the barrier between platinum and amorphous silicon. Such large dark leakage current is likely to play a role on defining the $E_{1 / 2}$ position for the ferrocene/ferricenium couple. This is because OCP values of photodiode can be proportionally affected by the dark leakage current. ${ }^{18}$ Therefore, even though the work function of $\mathrm{Pt}(-5.35 \mathrm{eV})$ is higher than the Fermi level of $\mathrm{p}^{+} \mathrm{Si}(100)$ (Table S1), E1/2 observed for ferrocene on $\mathrm{Pt} \mid \mathrm{aSi}(+0.081 \mathrm{~V})$ is less positive than on $\mathrm{p}^{+} \mathrm{Si}(100) \mid \mathrm{aSi}$ itself.

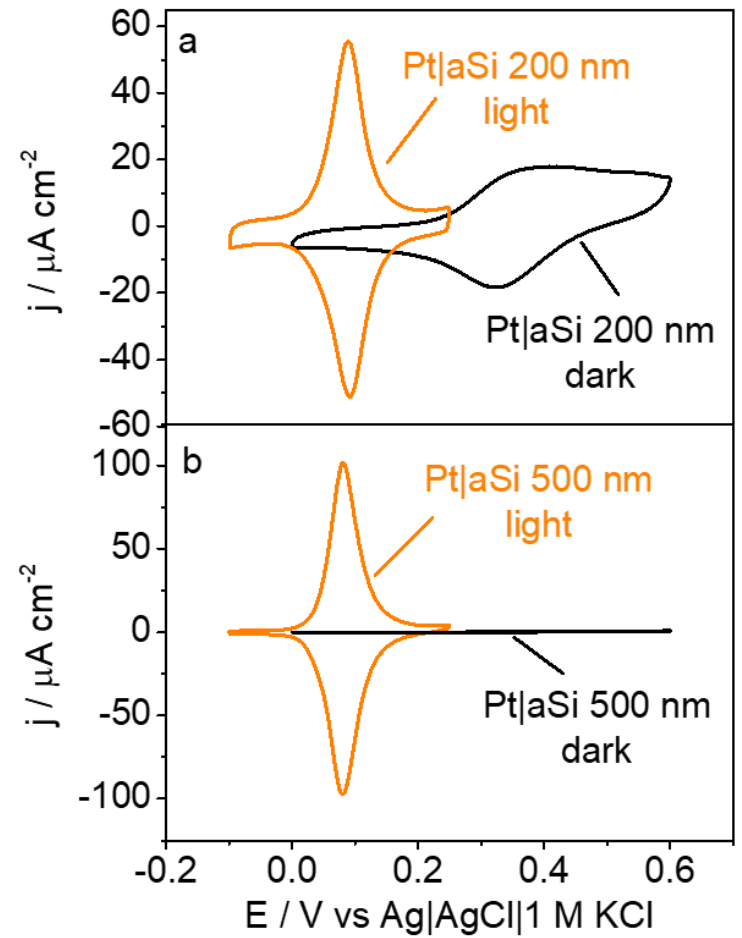

Figure 3 - Cyclic voltammograms of a ferrocene redox probe attached onto the monolayer-protected amorphous silicon. Photoactive amorphous silicon deposited onto Pt with thicknesses of (a) $200 \mathrm{~nm}$ and (b) $500 \mathrm{~nm}$. Electrolyte $=1 \mathrm{M}$
$\mathrm{HClO}_{4} ; v=100 \mathrm{mV} \mathrm{s}^{-1}$; surface topside illuminated with supra bandgap white light at $94 \mathrm{~mW} \mathrm{~cm}^{-2}$.

The results presented above show how to control the peak position of a model redox monolayer at a constant light intensity condition. Note, for that, electrochemical pre-treatment is required. The pre-treatment consists of cycling the ferrocene modified electrodes in $1 \mathrm{M} \mathrm{HClO}_{4}$ for 30 cycles (See the change in the CVs for a $\mathrm{p}^{+} \mathrm{Si}(100) \mid \mathrm{aSi}$ surface in Figure 4). During the initial cycles, the position of Epa appears at potentials more negative than $\mathrm{Ep}_{\mathrm{c}}$ and $\Delta \mathrm{Ep}<0 \mathrm{~V}$. The reason for a peak inversion in ferrocene-modified semiconducting electrodes was previously reported as a manifestation of attractive electrostatic forces between the positive ferricenium moieties and photoelectrons. ${ }^{43}$ Mainly, the static positive charges of the electrochemical generated ferricenium moieties is believed to lift the electronic bands of the semiconductor and distort the magnitude of the band bending at the semiconductor-electrolyte interface. The consequence is an increase of reduction rates and a decrease of oxidation rates that leads to the inversion phenomena. The question that arises here is what is a possible cause of this dynamic behavior that leads to the loss of peak inversion? As seen in Figure 4, within the first 20 cycles, $\mathrm{Ep}_{\mathrm{a}}$ shifts relatively quick towards $\mathrm{Ep}_{\mathrm{c}}$. After the $30^{\text {th }}$ cycle, the peak inversion at $100 \mathrm{mV} \mathrm{s}^{-1}$ disappears and the voltammogram tends to stabilize. All data discussed here was acquired after performing this protocol.

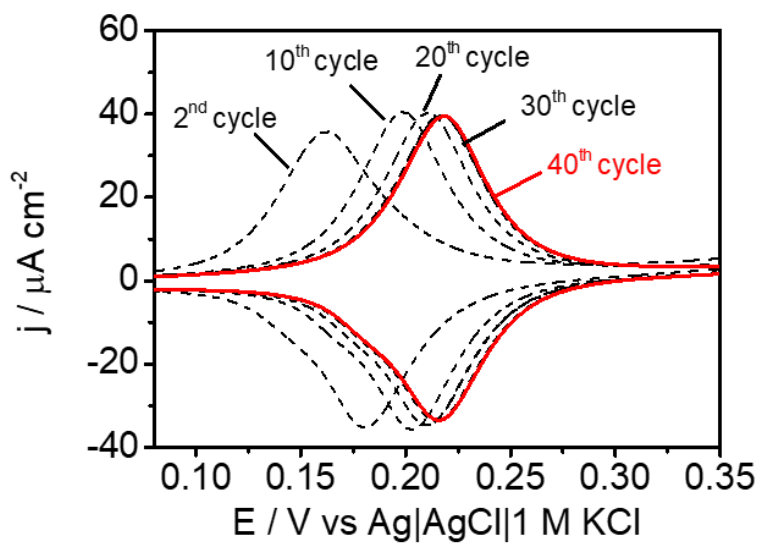

Figure 4 - Initial cycles at $100 \mathrm{mV} \mathrm{s}^{-1}$ of a ferrocene redox probe attached onto $\mathrm{p}^{+} \mathrm{Si}(100) \mid \mathrm{aSi} 200 \mathrm{~nm}$ photoelectrode. Electrolyte = $1 \mathrm{M} \mathrm{HClO}_{4} ; \mathrm{v}=100 \mathrm{mV} \mathrm{s}^{-1}$; surface topside illuminated with supra bandgap white light at $94 \mathrm{~mW} \mathrm{~cm}^{-2}$.

In an attempt to answer this question, XPS was performed on the $\mathrm{p}^{+} \mathrm{Si}(100) \mid \mathrm{aSi}$ system to monitor the evolution of $\mathrm{SiO}_{\mathrm{x}}$ levels after the click-attachment of ferrocene moieties and after performing 30 voltammetric cycles. This is because an increase in the amount of an insulating $\mathrm{SiO}_{\mathrm{x}}$ layer could mask the inversion of the peaks by reducing electron-transfer rates. ${ }^{43}$ As shown in Figure 5, the percentage of $\mathrm{SiO}_{\mathrm{x}}$ on the surface [Area $\mathrm{SiO}_{x} 2 p /\left(\right.$ Area $\left.\mathrm{SiO}_{x} 2 p+\mathrm{Si} 2 p_{3 / 2}\right)$ ] after coupling the ferrocene species was $4.6 \%$. The $\mathrm{SiO}_{\mathrm{x}}$ level registered after 30 cycles was $5.3 \%$. The similarities between 
these results suggests that changes in $\mathrm{SiO}_{\mathrm{x}}$ levels are not responsible by the disappearance of the peak inversion. Therefore, it is hypothesized that: (i) within the initial cycles the peak inversion disappears because the electronic bands of amorphous silicon become pinned by the existence of a small amount of $\mathrm{SiO}_{\mathrm{x}}{ }^{44}$; (ii) and/or the concentration of charge-carriers in the amorphous layer changes during the initial cycles and an electrochemical pre-treatment is required to stabilize its electronic properties.

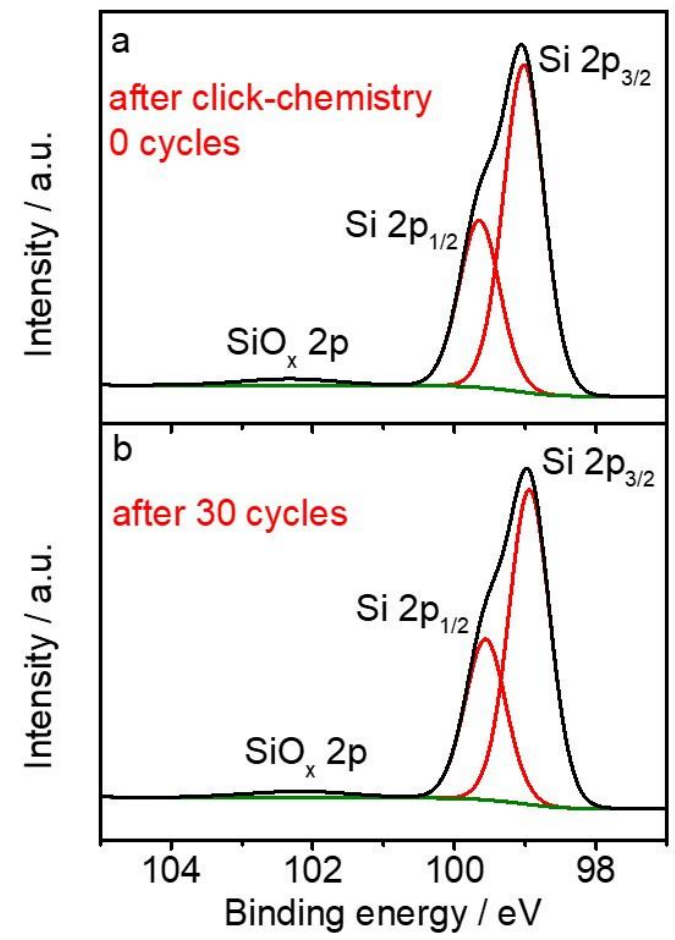

Figure 5 - XPS Si 2p narrow scan of p+Si(100)|aSi surfaces after click-attachment of azidomethylferrocene and after 30 voltammetric cycles in $1 \mathrm{M} \mathrm{HClO}_{4}$.

Finally, Mott-Schottky plots were recorded to understand in which potential range, processes of electron-transfer are possible to be tuned with heterojunctions. As shown in Figure $\mathrm{S} 4$, flat-band potentials $\left(\mathrm{E}_{\mathrm{FB}}\right.$ ) were determined as $-1.8 \mathrm{~V}$ for $\mathrm{n}^{+} \mathrm{Si}(100) \mid \mathrm{aSi},-1.15 \mathrm{~V}$ for Pt|aSi and $-0.95 \mathrm{~V}$ for ITO|aSi. Surprisingly, for the $\mathrm{p}^{+} \mathrm{Si}(100) \mid \mathrm{aSi}$ surface, the dark electrode-electrolyte interface remained depleted of charge carriers between $-2 \mathrm{~V}$ to $+1 \mathrm{~V}$. These results imply that any redox monolayer that can be monitored in aqueous-based electrolytes can be interrogated on these amorphous silicon-based heterojunction electrodes. This is contrary to what one observes with other semiconducting electrodes where the doping level must be chosen depending on the redox potential of the species of interest. ${ }^{3}$

This finding motivated us to modify amorphous silicon with a mixed redox self-assembled monolayer prepared with both azidomethylferrocene and azidomethylanthracene9,10-dione. Without the use of heterojunctions, photomodulation of ferrocene needs to be performed on a n-type silicon, ${ }^{19}$ while anthracene-9,10-dione molecules neces- sarily requires a p-type electrode. ${ }^{20}$ This is because photoeffects are generally not observed at accumulated n-type and p-type electrodes for redox couples located at potentials negative and positive of their flat-band potential, respectively. ${ }^{45}$ As shown in Figure 6 a for $\mathrm{p}^{+} \mathrm{Si}(100) \mid \mathrm{aSi}$, the use of heterojunctions allowed us to observe redox peaks for both ferrocene and anthracene-9,10-dione on a single silicon-type electrode. That is, the existence of a heterojunction expands the potential range suitable for photomodulation of redox species. Such capability could have practical implications for sensors, photocatalysis and platforms for electrochemical mapping and electrochemical patterning. $32,38,46$ Additionally, as shown in Figure 6b, the replacement of $\mathrm{p}^{+} \mathrm{Si}(100) \mid \mathrm{aSi}$ by an ITO|aSi electrode displaced the $\mathrm{E}_{1 / 2}$ of both redox processes to more negative potentials. Such modulation of the $E_{1 / 2}$ values follow the same trend predicted by Scheme 2 and discussed in Figure 1 for the redox self-assembled monolayers based exclusively on ferrocene.

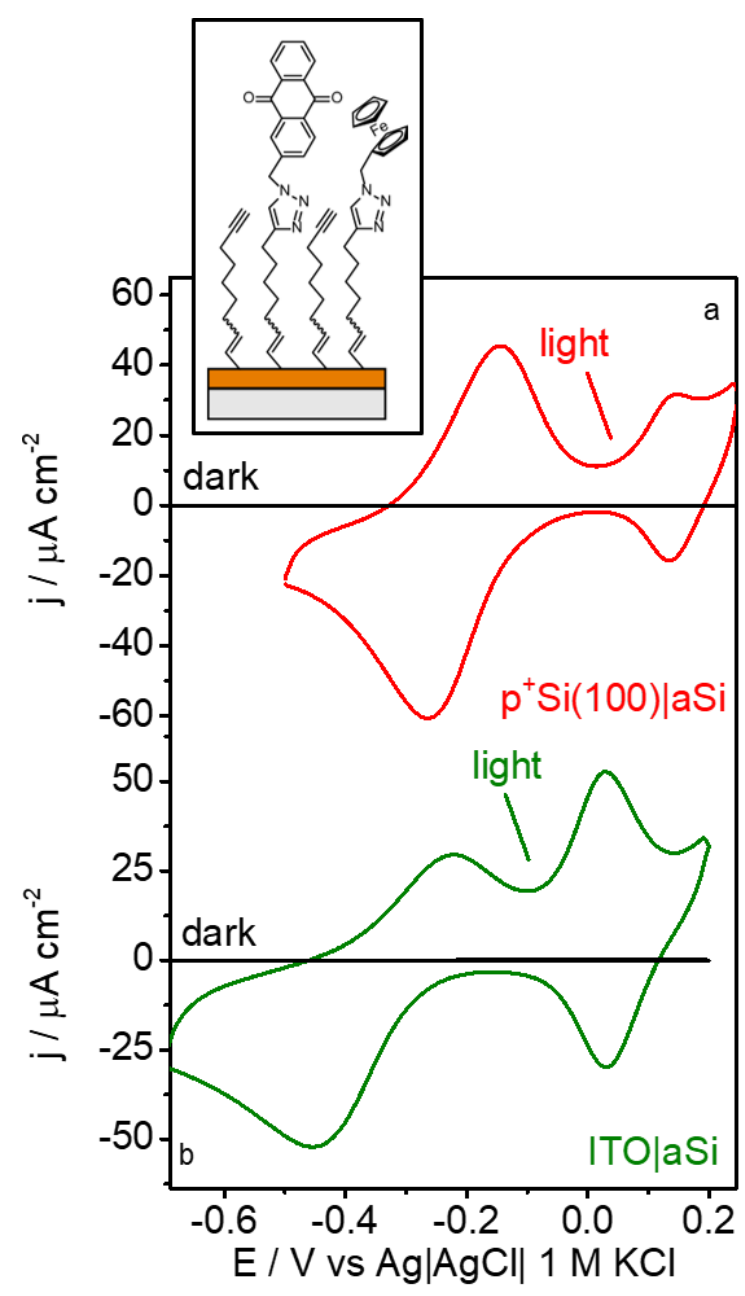

Figure 6 - Cyclic voltammetry at $500 \mathrm{mV} \mathrm{s}^{-1}$ in light and dark of a mixed redox SAM formed by anthracene-9,10-dione and ferrocene covalently attached to (a) $\mathrm{p}^{+} \mathrm{Si}(100) \mid \mathrm{aSi}$ and (b) ITO|aSi. Electrolyte $=1 \mathrm{M} \mathrm{NaClO}_{4}+25 \mathrm{mM} \mathrm{NaH}_{2} \mathrm{PO}_{4}$ $(\mathrm{pH}=11)$. Argon was bubbled during $30 \mathrm{~min}$ prior to cyclic voltammetry, with blanket kept on the electrolyte surface during measurements; surface topside illuminated with su-

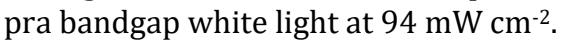




\section{CONCLUSION}

A surface engineering strategy for controlling peak positions of redox monolayers at a constant light-intensity is proposed. This was achieved by exploring the principle that contact of materials in heterojunctions tends to create a common equilibrium Fermi level for both sides of the interface. In addition to the large potential range on which heterojunctions were capable to operate, this work provides a helpful capability for the design of more versatile photoelectrodes, on which a great variety of redox monolayers can be photomodulated on a unique silicon-type surface. Such results could have practical implications for sensors, bioactive surfaces, catalytic devices and for the development of light-addressable platforms. ${ }^{47}$

\section{ASSOCIATED CONTENT}

Supporting Information. XPS evidence of surface-chemistry formation, table with electronic parameters of amorphous silicon and bulk conductors, band diagrams for each semiconducting heterojunctions, cyclic voltammogram of ferrocene attached to monolayer protected $\mathrm{n}-\mathrm{Si}(100)|\mathrm{aSi}, \mathrm{p}-\mathrm{Si}(100)| \mathrm{aSi}$ and glass|aSi, Mott-Schottky plots for each heterojunction. This material is available free of charge via the Internet at http://pubs.acs.org.

\section{AUTHOR INFORMATION}

${ }^{\S}$ Equal contribution

\section{Corresponding Authors}

*justin.gooding@unsw.edu.au

*v.goncales@unsw.edu.au

\section{ACKNOWLEDGMENT}

We acknowledge the financial support from the Australian Research Council Centre of Excellence in Convergent Bio-Nano Science and Technology (J.J.G CE14100036) and the ARC Australian Laureate Fellowship (J.J.G. FL150100060).

\section{REFERENCES}

1. Bard, A. J., Design of Semiconductor Photoelectrochemical Systems for Solar Energy Conversion. The Journal of Physical Chemistry 1982, 86, 172-177.

2. Sun, K.; Shen, S.; Liang, Y.; Burrows, P. E.; Mao, S. S.; Wang, D., Enabling Silicon for Solar-Fuel Production. Chemical Reviews 2014, 114, 8662-8719.

3. Choudhury, M. H.; Ciampi, S.; Yang, Y.; Tavallaie, R.; Zhu, Y.; Zarei, L.; Gonçales, V. R.; Gooding, J. J., Connecting Electrodes with Light: One Wire, Many Electrodes. Chemical Science 2015, 6, 6769-6776.

4. $\quad$ Zarei, L.; Tavallaie, R.; Choudhury, M. H.; Parker, S. G.; Bakthavathsalam, P.; Ciampi, S.; Gonçales, V. R.; Gooding, J. J., DNA-Hybridization Detection on Si(100) Surfaces Using LightActivated Electrochemistry: A Comparative Study between Bovine Serum Albumin and Hexaethylene Glycol as Antifouling Layers. Langmuir 2018, 34, 14817-14824.

5. Zhao, W.-W.; Xu, J.-J.; Chen, H.-Y., Photoelectrochemical Immunoassays. Analytical Chemistry 2018, 90, 615-627.

6. Parker, S. G.; Yang, Y.; Ciampi, S.; Gupta, B.; Kimpton, K.; Mansfeld, F. M.; Kavallaris, M.; Gaus, K.; Gooding, J. J., A
Photoelectrochemical Platform for the Capture and Release of Rare Single Cells. Nature Communications 2018, 9, 2288.

7. Ong, S. W. D.; Lin, J.; Seebauer, E. G., Control of Methylene Blue Photo-Oxidation Rate over Polycrystalline Anatase Tio2 Thin Films Via Carrier Concentration. The Journal of Physical Chemistry C 2015, 119, 11662-11671.

8. $\quad$ Liu, G.; Wang, L.; Yang, H. G.; Cheng, H.-M.; Lu, G. Q., Titania-Based Photocatalysts - Crystal Growth, Doping and Heterostructuring. Journal of Materials Chemistry 2010, 20, 831843.

9. Chen, S.; Wang, L.-W., Thermodynamic Oxidation and Reduction Potentials of Photocatalytic Semiconductors in Aqueous Solution. Chemistry of Materials 2012, 24, 3659-3666.

10. Anderson, N. C.; Carroll, G. M.; Pekarek, R. T.; Christensen, S. T.; van de Lagemaat, J.; Neale, N. R., Silicon Photoelectrode Thermodynamics and Hydrogen Evolution Kinetics Measured by Intensity-Modulated High-Frequency Resistivity Impedance Spectroscopy. The Journal of Physical Chemistry Letters 2017, 8, 5253-5258.

11. Wang, H.; Zhang, L.; Chen, Z.; Hu, J.; Li, S.; Wang, Z.; Liu, J.; Wang, X., Semiconductor Heterojunction Photocatalysts: Design, Construction, and Photocatalytic Performances. Chemical Society Reviews 2014, 43, 5234-5244.

12. Linford, M. R.; Chidsey, C. E. D., Alkyl Monolayers Covalently Bonded to Silicon Surfaces. Journal of the American Chemical Society 1993, 115, 12631-12632.

13. Ciampi, S.; Eggers, P. K.; Le Saux, G.; James, M.; Harper, J. B.; Gooding, J. J., Silicon (100) Electrodes Resistant to Oxidation in Aqueous Solutions: An Unexpected Benefit of Surface Acetylene Moieties. Langmuir 2009, 25, 2530-2539.

14. Gonçales, V. R.; Wu, Y.; Gupta, B.; Parker, S. G.; Yang, Y.; Ciampi, S.; Tilley, R.; Gooding, J. J., Stability of Chemically Passivated Silicon Electrodes in Aqueous Solutions: Interplay between Bias Voltage and Hydration of the Electrolyte. The Journal of Physical Chemistry C 2016, 120, 15941-15948.

15. Ciampi, S.; Böcking, T.; Kilian, K. A.; James, M.; Harper, J. B.; Gooding, J. J., Functionalization of AcetyleneTerminated Monolayers on $\mathrm{Si}(100)$ Surfaces: A Click Chemistry Approach. Langmuir 2007, 23, 9320-9329.

16. Yang, Y.; Cuartero, M.; Gonçales, V. R.; Gooding, J. J.; Bakker, E., Light-Addressable Ion Sensing for Real-Time Monitoring of Extracellular Potassium. Angewandte Chemie International Edition 2018, 57, 16801-16805.

17. Santangelo, P. G.; Miskelly, G. M.; Lewis, N. S., Cyclic Voltammetry at Semiconductor Photoelectrodes. 1. Ideal SurfaceAttached Redox Couples with Ideal Semiconductor Behavior. The Journal of Physical Chemistry 1988, 92, 6359-6367.

18. Vogel, Y. B.; Molina, A.; Gonzalez, J.; Ciampi, S., Quantitative Analysis of Cyclic Voltammetry of Redox Monolayers Adsorbed on Semiconductors: Isolating Electrode Kinetics, Lateral Interactions, and Diode Currents. Analytical Chemistry 2019, 91, 5929-5937.

19. Yang, Y.; Ciampi, S.; Gooding, J. J., Coupled Thermodynamic and Kinetic Changes in the Electrochemistry of Ferrocenyl Monolayers Induced by Light. Langmuir 2017, 33, 2497-2503.

20. Yang, Y.; Ciampi, S.; Choudhury, M. H.; Gooding, J. J., Light Activated Electrochemistry: Light Intensity and $\mathrm{Ph}$ Dependence on Electrochemical Performance of Anthraquinone Derivatized Silicon. The Journal of Physical Chemistry C 2016, 120, 2874-2882.

21. Battaglia, C.; Cuevas, A.; De Wolf, S., High-Efficiency Crystalline Silicon Solar Cells: Status and Perspectives. Energy \& Environmental Science 2016, 9, 1552-1576.

22. Jensen, N.; Hausner, R. M.; Bergmann, R. B.; Werner, J. H.; Rau, U., Optimization and Characterization of Amorphous/Crystalline Silicon Heterojunction Solar Cells. 
Progress in Photovoltaics: Research and Applications 2002, 10, 1 13.

23. Kanevce, A.; Metzger, W. K., The Role of Amorphous Silicon and Tunneling in Heterojunction with Intrinsic Thin Layer (Hit) Solar Cells. Journal of Applied Physics 2009, 105, 094507.

24. Masuko, K., et al., Achievement of More Than 25\% Conversion Efficiency with Crystalline Silicon Heterojunction Solar Cell. IEEE Journal of Photovoltaics 2014, 4, 1433-1435.

25. Mews, M.; Liebhaber, M.; Rech, B.; Korte, L., Valence Band Alignment and Hole Transport in Amorphous/Crystalline Silicon Heterojunction Solar Cells. Applied Physics Letters 2015, 107, 013902.

26. Su, J.; Li, G.-D.; Li, X.-H.; Chen, J.-S., 2d/2d Heterojunctions for Catalysis. Adv Sci (Weinh) 2019, 6, 18017021801702 .

27. Low, J.; Yu, J.; Jaroniec, M.; Wageh, S.; Al-Ghamdi, A. A., Heterojunction Photocatalysts. Advanced Materials 2017, 29, 1601694.

28. Leibovitch, M.; Kronik, L.; Fefer, E.; Korobov, V.; Shapira, Y., Constructing Band Diagrams of Semiconductor Heterojunctions. Applied Physics Letters 1995, 66, 457-459.

29. Orlowski, B. A.; Pieniazek, A.; Goscinski, K.; Kopalko, K., Quasi Fermi Levels in Semiconductor Photovoltaic Heterojunction. Acta Physica Polonica A 2016, 129, A-100-A-102. 30. Nandakumar, N. K.; Seebauer, E. G., Manipulating Reaction Rates of Metal-Oxide Heterogeneous Catalysts Via Semiconductor Heterojunctions. The Journal of Physical Chemistry C 2018, 122, 16655-16663.

31. Nandakumar, N. K.; Seebauer, E. G., Manipulating Surface Potentials of Metal Oxides Using Semiconductor Heterojunctions. The Journal of Physical Chemistry C 2016, 120, 5486-5494.

32. Lian, J.; Yang, Y.; Wang, W.; Parker, S. G.; Gonçales, V. R.; Tilley, R. D.; Gooding, J. J., Amorphous Silicon on Indium Tin Oxide: A Transparent Electrode for Simultaneous Light Activated Electrochemistry and Optical Microscopy. Chemical Communications 2019, 55, 123-126.

33. Ciampi, S.; Le Saux, G.; Harper, J. B.; Gooding, J. J., Optimization of Click Chemistry of Ferrocene Derivatives on Acetylene-Functionalized Silicon(100) Surfaces. Electroanalysis 2008, 20, 1513-1519.

34. Ahmad, S. A. A.; Ciampi, S.; Parker, S. G.; Gonçales, V. R.; Gooding, J. J., Forming Ferrocenyl Self-Assembled Monolayers on $\mathrm{Si}(100)$ Electrodes with Different Alkyl Chain Lengths for Electron Transfer Studies. ChemElectroChem 2019, 6, 211-220.

35. Nazarov, A. N.; Vovk, Y. N.; Lysenko, V. S.; Turchanikov, V. I.; Scryshevskii, V. A.; Ashok, S., Carrier
Transport in Amorphous Sic/Crystalline Silicon Heterojunctions. Journal of Applied Physics 2001, 89, 4422-4428.

36. Bard, A. J., Photoelectrochemistry and Heterogeneous Photo-Catalysis at Semiconductors. Journal of Photochemistry 1979, 10, 59-75.

37. Salvador, P., Semiconductors' Photoelectrochemistry: A Kinetic and Thermodynamic Analysis in the Light of Equilibrium and Nonequilibrium Models. The Journal of Physical Chemistry $B$ 2001, 105, 6128-6141.

38. Vogel, Y. B.; Gonçales, V. R.; Al-Obaidi, L.; Gooding, J. J.; Darwish, N.; Ciampi, S., Nanocrystal Inks: Photoelectrochemical Printing of $\mathrm{Cu} 20$ Nanocrystals on Silicon with 2d Control on Polyhedral Shapes. Advanced Functional Materials 2018, 28, 1804791.

39. Abdulraheem, Y.; Gordon, I.; Bearda, T.; Meddeb, H.; Poortmans, J., Optical Bandgap of Ultra-Thin Amorphous Silicon Films Deposited on Crystalline Silicon by Pecvd. AIP Advances 2014, 4, 057122.

40. Allan, G.; Delerue, C.; Lannoo, M., Electronic Structure and Localized States in a Model Amorphous Silicon. Physical Review B 1998, 57, 6933-6936.

41. Morigaki, K.; Ogihara, C., Amorphous Semiconductors: Structure, Optical, and Electrical Properties. In Springer Handbook of Electronic and Photonic Materials, Kasap, S.; Capper, P., Eds. Springer International Publishing: Cham, 2017; pp 1-1.

42. $\quad$ Ciampi, S.; James, M.; Choudhury, M. H.; Darwish, N. A.; Gooding, J. J., The Detailed Characterization of Electrochemically Switchable Molecular Assemblies on Silicon Electrodes. Physical Chemistry Chemical Physics 2013, 15, 98799890.

43. Vogel, Y. B., et al., Reproducible Flaws Unveil Electrostatic Aspects of Semiconductor Electrochemistry. Nature Communications 2017, 8, 2066.

44. Mizsei, J., Fermi-Level Pinning and Passivation on the Oxide-Covered and Bare Silicon Surfaces and Interfaces. Vacuum 2002, 67, 59-67.

45. Fabre, B., Functionalization of Oxide-Free Silicon Surfaces with Redox-Active Assemblies. Chemical Reviews 2016, 116, 4808-4849.

46. Vogel, Y. B.; Gonçales, V. R.; Gooding, J. J.; Ciampi, S., Electrochemical Microscopy Based on Spatial Light Modulators: A Projection System to Spatially Address Electrochemical Reactions at Semiconductors. Journal of The Electrochemical Society 2018, 165, H3085-H3092.

47. Vogel, Y. B.; Gooding, J. J.; Ciampi, S., LightAddressable Electrochemistry at Semiconductor Electrodes: Redox Imaging, Mask-Free Lithography and Spatially Resolved Chemical and Biological Sensing. Chemical Society Reviews 2019, 48, 37233739 .

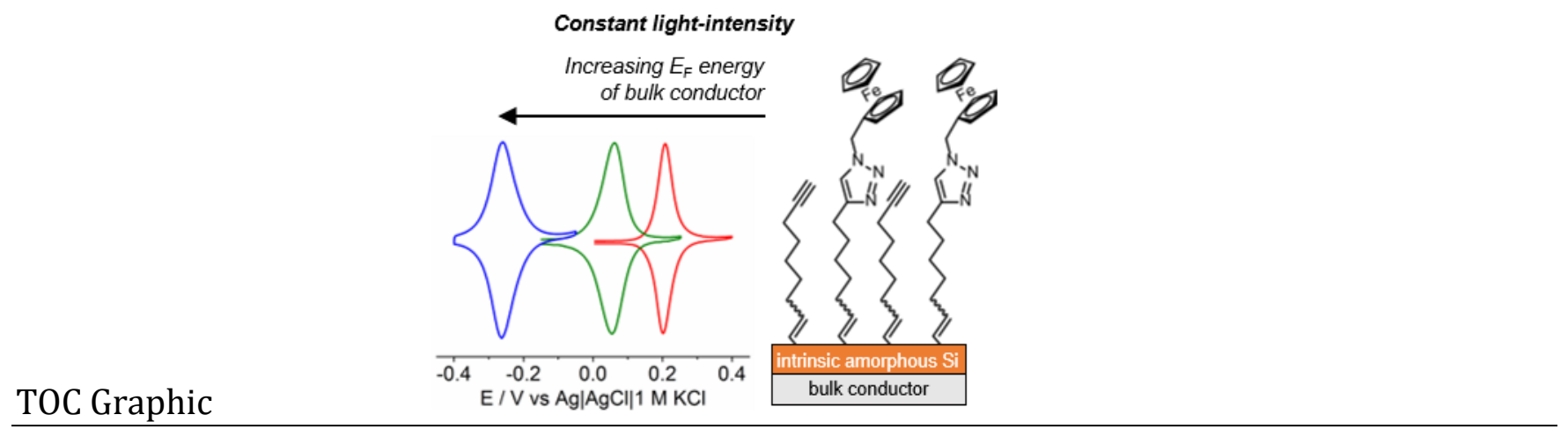

